\title{
An Elementary Algorithm for Autonomous Air Terminal Merging and Interval Management
}

\author{
Allan L. White ${ }^{1}$ \\ NASA Langley, Hampton, VA 23681
}

\begin{abstract}
A central element of air traffic management is the safe merging and spacing of aircraft during the terminal area flight phase. This paper derives and examines an algorithm for the merging and interval managing problem for Standard Terminal Arrival Routes. It describes a factor analysis for performance based on the distribution of arrivals, the operating period of the terminal, and the topology of the arrival routes; then presents results from a performance analysis and from a safety analysis for a realistic topology based on typical routes for a runway at Phoenix International Airport. The heart of the safety analysis is a statistical derivation on how to conduct a safety analysis for a local simulation when the safety requirement is given for the entire airspace
\end{abstract}

\section{INTRODUCTION}

This study develops and examines an algorithm for the merging and interval managing problem for Standard Terminal Arrival Routes (STARS). Once the algorithm is derived, there are two parts to this study. The first part consists of a factor analysis for performance that considers the distribution of arrivals, the operating period of the terminal, and the topology of the arrival routes. The second part conducts a performance analysis and a safety analysis for a realistic topology based on the routes for one of the runways at Phoenix International Airport.

The overall objective is to increase understanding of air traffic by creating algorithms and models. The merging and interval management of aircraft in a terminal appears to be a topic that can evolve into other areas. Secondary objectives include:

- Simulation for part of or all of the national airspace - such simulations can contribute to more efficient and safer routing and operations, and such simulations depend on accurate and efficient models of terminal operations.

- Operation of autonomous aircraft - autonomous aircraft can perform routine and boring tasks such as monitoring a forest for fires, and they can perform dangerous tasks such as crop dusting. We are more willing to send an autonomous craft instead of a piloted airplane into hazardous conditions. In the near future, we may see the autonomous operation of cargo carriers.

- Layout of arrival routes - there may or may not be two different criteria. One is the efficient and safe conduction of autonomous procedures. The other is to ease the burden of an air traffic controller. For air traffic controllers, our conjecture is that easing the burden leads to more efficient and safer operation.

- Extension to traffic outside the terminal area - in the material below, when an aircraft arrives at the terminal area, the algorithm computes whether or not it can enter the queue. One possibility is the converse of this where the algorithm computes the range of arrival speeds and arrival times that allow it to enter the queue. This can be applied to aircraft in transit. It can possibly be extended to cover the entire flight including take-off time.

Finally, there is the challenge of replacing pilots and controllers with algorithms. Work in this area can be the most fruitful endeavor even if autonomous algorithms are far in the future or are never implemented since developing an algorithm requires a deep understanding of a process. An example of this is a question that arises for texting. Currently, merging and spacing is performed with a multi-step sequence of adjustments whereas the algorithm below uses a single-step adjustment. A conjecture is that the algorithm below is too complex for a controller to compute in real time while a multi-step approach is a procedure within human capability. Is this conjecture correct? If so, what is an optimum procedure for air traffic controllers? This is a first effort, and much work remains to be done before it can contribute to any of the above goals.

The second section reviews the literature. The third section gives the assumptions for the algorithm. The fourth section discusses generalizing the assumptions. The fifth section introduces aircraft deceleration and the flight trajectory used by the algorithm. The sixth section derives the algorithm which considers whether an aircraft can enter an arrival route or must go into a holding pattern (based on whether or not an aircraft can maintain separation if it enters), and whether the flight trajectory of an aircraft is accepted. The seventh section presents the two arrival-route layouts used for the factor analysis of Standard Terminal Arrival Route topology and the performance results for these two layouts. The eighth section presents the layout based on the Phoenix International Airport along with a performance result. For this realistic layout, the ninth section changes from studying performance to studying safety. Since any safety requirement is likely to be stated for the global airspace, an important element is the statistical analysis of what a local simulation must demonstrate. The tenth section contains a summary of findings 
whereby the simple algorithm developed in this paper was shown to be effective and to have safely and efficiently handled all conditions of the considered air traffic scenarios.

There are many factors left unexamined, but we decided it was better to increase the realism before proceeding further.

\section{LITERATURE SURVEY}

In the paper [1], simulations are performed to study the gain in efficiency by combining continuous-descent and airborneprecision-spacing. No merging is considered. In the scenarios, there was loss of separation. Another paper [2] presents concepts for robust operations in the face of uncertainties. Future simulations for the concepts are planned. The paper [3] develops a merging and spacing algorithm. Trials have been conducted, and more are planned, but the paper does not list any of the results. The paper [4] attempts an autonomous system that performs conflict resolution and arrival scheduling for arrivals and departures at the Dallas/Fort Worth International Airport and the Dallas Love Field Airport. This paper, however, states it was not successful in its attempt to devise an autonomous algorithm. The paper [5] derives sufficient conditions on incoming aircraft spacing and set of arrival times to guarantee feasible arrival times at a merge point. The method is extended to more general scenarios by means of a binary tree. The paper [6] studies the efficient merging for a pair of aircraft in the presence of uncertainty. The paper [7] also studies the spacing problem for pairs of aircraft given the lack of accurate trajectory information.

In contrast, the algorithm below makes it possible to conduct a performance analysis for an entire Standard Terminal Arrival Route and to conduct a global analysis for safety.

\section{ASSUMPTIONS}

As a first effort, there are a number of simplifying assumptions:

- There is only one runway, and only arrivals are considered.

- The algorithm applies from the Entry-Points of the arrival routes to the Waypoint where the aircraft begins its descent to land.

- The distance from all the Entry Points to the Waypoint is the same, in this case 50 nautical miles. This allows a firstcome-first-served policy.

- All aircraft are the same. They arrive at the Entry Points at 250 knots and slow to 150 knots at the Waypoint. They all have the same rate of deceleration, and the spacing requirement is 5 nautical miles.

- When an aircraft arrives at an Entry Point, it is either accepted or rejected. If accepted, the algorithm computes its flight plan. If rejected, it goes into a holding pattern which, for this study, is a simple maneuver. The aircraft flies a circle turning at the rate of one degree per second and arrives at the same Entry Point six minutes later where the algorithm, once again, either accepts or rejects it.

- The aircraft follow the routes precisely. There is no flight perturbation or navigation error. In addition, the aircraft remain at the same altitude.

\section{Generalizing the Assumptions}

This paper is far from a finished product because of the current assumptions. This section will discuss their generalization.

Only arrivals are considered because departures are under control of the airport and handling arrivals is more demanding. Worthwhile extensions are to consider multiple runways at the same airport and the routing problem for airports in close proximity.

The route from the Entry Points to the Waypoints where the aircraft began their descent is the critical part. There are studies for the descent $[8,9]$.

Items to study include the equal-distance assumptions and the first-come-first-served policy. First, however, note that this policy will work even if the distances from the Entry Points to the Waypoint are unequal, but it may become inefficient if there is a great difference. For instance, if one distance is 50 nautical miles and another is 20 , then aircraft arriving at the 20-nauticalmile point will most likely not be able to decelerate quickly enough to get behind an aircraft that has arrived at the 50-milepoint. The solution is an algorithm that can insert an aircraft between two previously arrived aircraft, but one can ask if this extension is worthwhile. At a busy airport, aircraft can arrive closely enough together that a chance to insert one aircraft between another two seldom arises. There is also the possibility that equal or nearly equal distances offer enough advantages for both algorithms and air traffic controllers that arranging such arrival routes is worthwhile. This factor remains to be examined. 
Generalizing to handling different classes is a reasonable extension. There are velocity restrictions on aircraft in the airspace of an airport. A research problem is examining what can be gained from handling different aircraft in a different manner. There may be a tradeoff between efficiency of a single aircraft versus efficiency of an ensemble of aircraft.

The holding pattern needs more work. Aircraft in a holding pattern are likely to fly at a different altitudes and their routes need to be specified. Instead of a fixed route, the route may be adjusted for efficient reentry into traffic and acceptance into the landing pattern.

Perturbations, errors, and decisions under uncertainty have been previously examined for separation assurance [10]. They can be included into merging and spacing. Having aircraft enter at different altitudes creates a three dimensional Standard Terminal Arrival Route. The advantages and disadvantages of this forms a research problem.

Related to generalizing the assumptions is applying optimization methods. This paper does not consider them for several reasons. First, this is an initial effort (by the author), and the goal was to derive an effective algorithm. Second, from the point of view of the airport, a measure of efficiency is how closely spaced the aircraft are, and for example below, the average spacing is 5.1 nautical miles at the Waypoint. Since the spacing must be at least 5 nautical miles and there are random arrivals, the current algorithm is efficient for this parameter. Third, the parameter for efficiency from the point of view of the aircraft is the average number of aircraft sent into a holding pattern, but a major factor for this parameter is the arrival distribution which is not part of a merging-and-spacing algorithm. An extension of the material in this paper, however, is using this algorithm to computer arrival windows for aircraft.

\section{DECELERATION AND AIRCRAFT TrAJECTORY}

A typical deceleration rate for a commercial aircraft is 10 to 15 knots per nautical mile [11]. Using v for velocity, $\mathrm{u}$ for distance, and $\mathrm{k}$ for the change in velocity per distance, we have

$$
\frac{\Delta v}{\Delta u}=-k
$$

Multiplying through by $\Delta \mathrm{u}$, dividing by $\Delta \mathrm{t}$, and letting $\Delta \mathrm{t}$ go to zero gives

$$
\mathrm{a}=\frac{\mathrm{d} \mathrm{v}(\mathrm{t})}{\mathrm{dt}}=-\mathrm{kv}(\mathrm{t})
$$

Hence, deceleration is proportional to velocity.

The above suggests an exponential decrease in velocity with respect to time, and this could be used, but this work will use constant deceleration for the aircraft trajectory. Taking the lower figures of 10 knots per nautical mile and the lower velocity of 150 knots gives a deceleration rate of 1500 knots per hour.

This study considers a simple trajectory for an aircraft that enters an arrival route. The aircraft proceeds at 250 knots to a deceleration point where it decelerates at 1500 knots per hour to a velocity of 150 knots. The time spent in deceleration is $1 / 15$ of an hour, and the distance traveled while decelerating is 200/15 nautical miles.

\section{REJECTION AND DECELERATION POINTS}

When an aircraft enters the terminal area, two computations are made. The first is whether the aircraft can proceed on an arrival-route to the Waypoint or if it will have to go into a holding pattern. The second is computing its deceleration point if it can proceed. This computation is based on the Entry Point, position, and flight condition of the previous aircraft.

The previous aircraft can have the same or different Entry Point. Its position is the distance it has traveled since entry. Its flight conditions are cruising at 250 knots, decelerating, or cruising at 150 knots.

The craft already on an arrival-route is known as the first craft. The craft entering is known as the second craft. The notation throughout this section is

$$
\begin{aligned}
& \mathrm{d}=\text { distance of first craft from its Entry Point } \\
& \mathrm{p}=\text { deceleration point of first craft } \\
& \mathrm{s}=\text { distance to junction for first craft } \\
& \mathrm{u}=\text { position of first craft when the second craft reaches } 150 \text { knots }
\end{aligned}
$$


The second craft must go into a holding pattern if it cannot decelerate quickly enough to maintain a 5 nautical mile lateral separation. The quickest the second craft can decelerate is immediately upon entering an arrival-route in which case it reaches 150 knots is in 1/15 of an hour during which time it has traveled 200/15 nautical miles. This will be the baseline for the holdingpattern decision.

\section{A. Same Entry Point Rejection Conditions}

We first consider holding-pattern conditions. The second craft goes into a holding pattern if $d<5$. Suppose $d$ otherwise.

Case 1: $\mathrm{d}<\mathrm{p}$

In this case, the first craft is going 250 knots when the second enters. If the second decelerates immediately upon its entry, then it will be 5 nautical miles or more behind the first and moving more slowly. Hence, there is no go-around.

Case 2: $\mathrm{p}<\mathrm{d}<\mathrm{p}+200 / 15$

In this case, during its deceleration phase, the second craft is going faster than the first craft. At the end of the first craft's deceleration phase, both craft are going 150 knots since the first craft will reach the end of its deceleration phase first. Hence, the separation is minimal at the end of the first craft's deceleration phase.

At time $1 / 15$ of an hour, the first craft is at position $(p+200 / 15)+150\left(1 / 15-\tau_{2}\right)$ where $\tau_{2}$ is the time needed for the first craft to complete its deceleration from d to $p+200 / 15$. Now

$$
\tau_{2}=\frac{1}{15}-\tau_{1}
$$

where $\tau_{1}$ is the time the first craft spends going from $\mathrm{p}$ to $\mathrm{d}$. This is given by

$$
\int_{0}^{\tau_{1}}(250-1500 t) d t=d-p
$$

or

$$
\tau_{1}=\frac{250-\sqrt{250(250)-4(750)(d-p)}}{1500}
$$

The craft goes into a holding pattern if

$$
p+\frac{200}{15}+150\left(\frac{1}{15}-\tau_{2}\right)<\frac{200}{15}+5
$$

or

$$
p+150 \tau_{1}<5
$$

Case 3: $d>p+200 / 15$

When the second craft finishes its immediate descent, it will be at 200/15, and the first craft will be at $d+150(1 / 15)=d+10$ $>p+200 / 15+10>200 / 15+5$. Hence, there will be no go-around.

\section{B. Same Entry Trajectory Computations}

We compute the deceleration point for the second craft assuming it is accepted.

Case 1: $\mathrm{d}<\mathrm{p}$

Consider the point at which the second craft reaches 150 knots. We wish it to be at least 5 nautical miles behind the first craft. If the first craft is going faster than 150 knots, then the first craft will pull ahead which leads to inefficient spacing. Hence, we want the second craft to reach 150 knots when the first is doing 150 knots and to be 5 nautical miles behind.

Let $\mathrm{p}_{2}$ be the deceleration point for the second craft and $\mathrm{u}$ be the position of the first craft when the second reaches $150 \mathrm{knots}$. Then we want

$$
p_{2}+\frac{200}{15}=u-5
$$

The time it take the second craft to reach 150 knots is $\tau$ where 


$$
\tau=\frac{p_{2}}{250}+\frac{1}{15}
$$

The relationship between $\tau$ and $\mathrm{d}, \mathrm{p}$, and $\mathrm{u}$ for the first craft is

$$
\tau=\frac{p-d}{250}+\frac{1}{15}+\frac{u-p-200 / 15}{150}
$$

Solving for $\mathrm{u}$ in equation (8) and substituting gives

$$
\frac{p_{2}}{250}+\frac{1}{15}=\frac{p-d}{250}+\frac{1}{15}+\frac{p_{2}+200 / 15+5-p-200 / 15}{150}
$$

which gives

$$
p_{2}=(15)(25)\left[\frac{p-5}{150}-\frac{p-d}{250}\right]
$$

Case 2: $\mathrm{p}<\mathrm{d}<\mathrm{p}+200 / 15$

As before, we want

$$
p_{2}+\frac{200}{15}=u-5
$$

and the time it takes the second craft to reach 150 knots is

$$
\tau=\frac{p_{2}}{250}+\frac{1}{15}
$$

The time it takes the first craft to complete its deceleration and travel to point $\mathrm{u}$ is

$$
\left(\frac{1}{15}-\tau_{1}\right)+\frac{u-(p+200 / 15)}{150}
$$

where $\tau_{1}$ is the time it takes the first craft to travel from $\mathrm{p}$ to $\mathrm{d}$, which, as before, is given by

$$
\int_{0}^{\tau_{1}}(250-1500 t) d t=d-p
$$

or

$$
\tau_{1}=\frac{250-\sqrt{250(250)-4(750)(d-p)}}{1500}
$$

Solve for $\mathrm{u}$ in equation (13), set equation (12) equal to $\tau$ and get

$$
p_{2}=(15)(25)\left[\frac{p-5}{150}+\tau_{1}\right]
$$

Case 3: $\mathrm{p}+200 / 15<\mathrm{d}$

As before, we want 


$$
p_{2}+\frac{200}{15}=u-5
$$

and the time it take the second craft to reach 150 knots is

$$
\tau=\frac{p_{2}}{250}+\frac{1}{15}
$$

The time it takes the first craft to travel to point $u$ is $(u-d) / 150$. Solving this set of equations gives

$$
p_{2}=\left(\frac{25}{15}\right)\left[d+5-\frac{200}{15}\right]
$$

\section{Different Entry Point Discussion}

For different Entry Points, craft can have nearly simultaneous arrival times, but the second craft may be able to decelerate quickly enough to maintain separation. (This leads to the conjectures that multiple Entry Points, greater distances before reaching a junction, and alternating Entry Points can improve efficiency.)

First observe that if the distance $d$ for the first craft is greater than 5 nautical miles, then the go-around and deceleration-point computations are the same as for the two craft having the same Entry Point. Hence, for the following, assume $d$ is less than 5 nautical miles.

For this initial effort, we assume that all the distances from any Entry Point to the first junction are greater than or equal to 20 nautical miles. These assumptions let the second craft decelerate to 150 knots and be at least 5 nautical miles from the junction.

The approach is to require the second craft to be 5 nautical miles behind the first craft when the first craft reaches the junction and to require the second craft to be traveling at 150 knots. Obviously, the second requirement creates some inefficiency, but our working hypothesis is that at a busy terminal, most craft will have to decelerate to 150 knots before they reach a junction which implies the inefficiency will be small.

\section{Different Entry Point Rejection Conditions}

The basis for deciding on a holding pattern is the maximum amount of time it takes for the second craft to immediately decelerate and reach five nautical miles before the junction. This expression is

$$
\frac{1}{15}+\frac{u-5-200 / 15}{150}
$$

If the first craft takes longer than this to reach the junction, the second craft must perform a go-around.

Case 1: $\mathrm{d}<\mathrm{u}<\mathrm{p}$

The first craft reaches the junction at time $(\mathrm{u}-\mathrm{d}) / 250$, and the second craft performs a go-around if this quantity is larger than the quantity in (20).

Case 2: $\mathrm{d}<\mathrm{p}<\mathrm{u}<\mathrm{p}+200 / 15$

The first craft reaches the junction at time

$$
\frac{p-d}{250}+\tau
$$

Where

$$
\int_{0}^{\tau}(250-1500 t) d t=u-p
$$

Or

$$
\tau=\frac{250-\sqrt{250(250)-4(750)(u-p)}}{1500}
$$


Go into holding pattern as per above.

Case 3: $\mathrm{d}<\mathrm{p}<\mathrm{p}+200 / 15<\mathrm{u}$

The first craft reaches the junction at time

$$
\frac{p-d}{250}+\frac{1}{15}+\frac{u-(p+200 / 15)}{150}
$$

The decision is made as before.

Case 4: $\mathrm{p}<\mathrm{d}<\mathrm{u}<\mathrm{p}+200 / 15$

Now $u \geq 20$ and $u<p+200 / 15$ implies $p>u-200 / 15$ which gives $d>p>20-200 / 15>5$. The decision is based on the same criterion as same Entry Point.

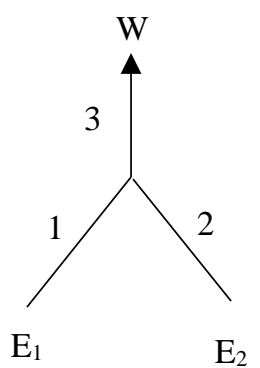

Figure 1: The Y Layout

Case 5: $\mathrm{p}<\mathrm{d}<\mathrm{p}+200 / 15<\mathrm{u}$

Note that $\mathrm{d}<5$ and the first craft is already decelerating. Hence, the second craft always has a velocity greater than or equal to the first, and the distance between them will always be less than 5 nautical miles. Perform a go-around.

Case 6: $\mathrm{p}<\mathrm{p}+200 / 15<\mathrm{d}$

Since $d>200 / 15>5$, this case is handled by the same-entry criterion.

\section{The ARrival Routes for Factor ANALYSIS}

Two layouts are considered for factor analysis. In both, all distances from any Entry Point to the Waypoint are equal to 50 nautical miles. In the Y layout in Fig. 1, segments and 2 are 30 nautical miles while segment 3 is 20 nautical miles. The Waypoint (where the aircraft begins its descent) is labeled W, and the Entry Points are labeled E.

In the Four Points layout in Fig. 2, segments 1 and 2 are 40 nautical miles, segments 3 and 4 are 30 nautical miles, and segments 5 and 6 are 10 nautical miles.

In the Four Points layout, the distance to a merging junction depends on the entry points of the two aircraft. Suppose the first aircraft enters at $E_{3}$. If the next aircraft enters at $\mathrm{E}_{4}$, the distance to merge is 30 nautical miles, but if the next aircraft enters at $\mathrm{E}_{1}$, the distance to merge is 40 nautical miles.

The tables below present two parameters for performance. One is average distance. When an aircraft reaches the Waypoint for descent, this parameter is the average distance of the following aircraft. This parameter indicates the efficiency of the airport: how much traffic it can handle. The second parameter is the average number of aircraft sent into a holding pattern per day. This reflects performance from the point of view of the aircraft since being sent into a holding pattern is an inconvenience.

The basic unit in all the simulations is one day of operations where 500 aircraft arrive. As mentioned before, we consider only one runway and only landings. All the simulations use a one second time increment for both arrival and aircraft movement. The factors considered are (1) arrival rate with perturbations, (2) distribution of Entry Point arrivals, and (3) number of Entry Points as displayed in the two different topologies.

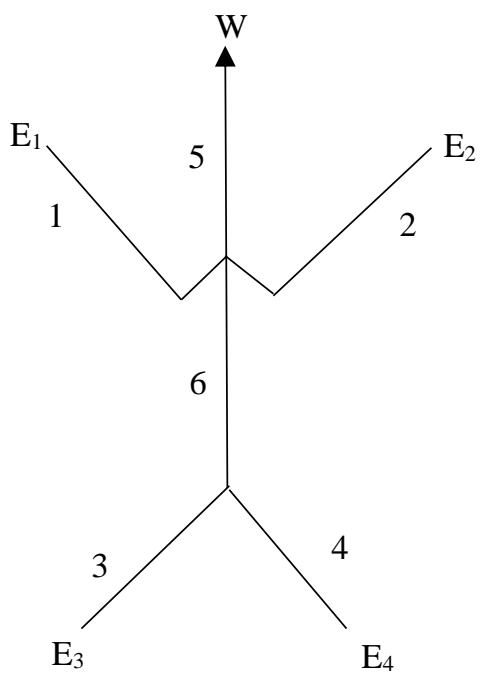

Figure 2: The Four Points Layout

The first arrival rate considered is based on terminal landing capacity. An interval of 5 nautical miles between aircraft going 150 knots implies a time interval of 120 seconds. The arrivals are originally set at 120 seconds apart and then perturbed. The perturbations have normal distributions with zero means and varying standard deviations. The standard deviations used are 0 , 30 and 60 seconds. For this arrival rate, the operating day for a terminal is about 16.6 hours.

As will be seen, the above arrival rate in conjunction with perturbations sends a number of aircraft into a holding pattern. Hence, an arrival time of 160 seconds with perturbations of 30 and 60 seconds was examined. This arrival rate gives an operating day of about 22 hours. 
Two distributions for location of arrival were considered. The first is that the Entry Point is chosen randomly with each Entry Point equally likely. The second is the aircraft alternate or rotate their arrival locations.

For each scenario, 365 daily runs are performed. Two statistics for each scenario are presented below. The first is the average Waypoint spacing which is the average distance between an aircraft when it reaches the Waypoint and the aircraft immediately following it. For each scenario, this statistic is collected per aircraft which implies a sample size of 500(365) $=182500$.

The second statistic is the average number of aircraft sent to a holding pattern per day. For each scenario, this statistics has a sample size of 365 . Note that an aircraft can be sent into a holding pattern more than once as it attempts to enter an arrival route.

The results are given plus or minus a $95 \%$ confidence interval, which seems adequate when considering performance criteria.

Table 1 gives the results for an average spacing of 120 seconds. One of the more dramatic statistics is the average number of aircraft sent into a holding pattern per day as the standard deviation increases. The explanation is the tight schedule. Originally, aircraft are arriving, on the average, as fast as the terminal can handle them. If perturbations cause a go-around, that aircraft joins the latter part of the arriving aircraft which implies the aircraft are now, on the average, arriving faster than the terminal can handle them. There is a cumulative effect.

Table 2 gives the results for an average spacing of 160 seconds. Comparing the two tables, the arrival spacing of 120 seconds is efficient with respect to airport operations but it is inefficient with respect to vehicles because of the number of aircraft sent into a holding pattern. The arrival spacing of 160 is less efficient with respect to airports because of the longer operation time, but it is more efficient with respect to aircraft especially if arrival at Entry Points is rotated.

\section{The Phoenix Layout}

This section considers a realistic example. Fig. 3 displays the layout for one of the runways at Phoenix International.

For this study, the lengths of the segments are rounded off to the nearest 5 nautical miles, and the lengths of segments 4, 5, 6, and 7 are adjusted so that the distance from any Entry Point to the Waypoint is 50 nautical miles. Segments 1 and 2 are 5 nautical miles; segments 3 , 4 , and 5 are 20 nautical miles; segment 6 is 45 nautical miles; and segment 7 is 40 nautical miles.
Table 1. Summary of results for an average spacing of 120 seconds.

\begin{tabular}{|c|l|l|}
\hline & Four Points & $\mathrm{Y}$ \\
\hline std $=0$ & & \\
\hline Avg Waypoint spacing & $5.28 \mathrm{e}+0 \pm 5.27 \mathrm{e}-10$ & $5.28 \mathrm{e}+0 \pm 5.27 \mathrm{e}-10$ \\
\hline Avg holding pattern & 0 & 0 \\
\hline & & \\
\hline std $=30$ & & $5.08 \mathrm{e}+0 \pm 2.26 \mathrm{e}-3$ \\
\hline Avg Waypoint spacing & $5.06 \mathrm{e}+0 \pm 1.53 \mathrm{e}-3$ & $3.67 \mathrm{e}+2 \pm 7.76 \mathrm{e}+0$ \\
\hline Avg holding pattern & $1.44 \mathrm{e}+2 \pm 7.37 \mathrm{e}+0$ & \\
\hline & & $5.11 \mathrm{e}+0 \pm 2.98 \mathrm{e}-3$ \\
\hline std $=60$ & & $5.79 \mathrm{e}+2 \pm 8.43 \mathrm{e}+0$ \\
\hline Avg Waypoint spacing & $5.08 \mathrm{e}+0 \pm 1.77 \mathrm{e}-3$ & \\
\hline Avg holding pattern & $3.02 \mathrm{e}+2 \pm 6.41 \mathrm{e}+0$ & \\
\hline & & $5.05 \mathrm{e}+0 \pm 1.56 \mathrm{e}-3$ \\
\hline std $=30 ;$ rotation & & $1.14 \mathrm{e}+2 \pm 4.67 \mathrm{e}+0$ \\
\hline Avg Waypoint spacing & $5.03 \mathrm{e}+0 \pm 1.02 \mathrm{e}-3$ & \\
\hline Avg holding pattern & $3.13 \mathrm{e}+1 \pm 3.72 \mathrm{e}+0$ & $5.09 \mathrm{e}+0 \pm 2.35 \mathrm{e}-3$ \\
\hline & & $4.62 \mathrm{e}+2 \pm 6.87 \mathrm{e}+0$ \\
\hline std $=60 ;$ rotation & & \\
\hline Avg Waypoint spacing & $5.07 \mathrm{e}+0 \pm 1.48 \mathrm{e}-3$ & \\
\hline Avg holding pattern & $2.40 \mathrm{e}+2 \pm 5.64 \mathrm{e}+0$ &
\end{tabular}

Table 2. Summary of results for an average spacing of 160 seconds

\begin{tabular}{|l|l|l|}
\hline & Four Points & $\mathrm{Y}$ \\
\hline std $=30$ & & \\
\hline Avg Waypoint spacing & $7.89 \mathrm{e}+0 \pm 9.80 \mathrm{e}-3$ & $7.86 \mathrm{e}+0 \pm 1.03 \mathrm{e}-2$ \\
\hline Avg holding pattern & $1.61 \mathrm{e}+0 \pm 1.96 \mathrm{e}-1$ & $5.94 \mathrm{e}+0 \pm 4.64 \mathrm{e}-1$ \\
\hline & & \\
\hline std $=60$ & & \\
\hline Avg Waypoint spacing & $7.65 \mathrm{e}+0 \pm 1.42 \mathrm{e}-2$ & $7.51 \mathrm{e}+0 \pm 1.47 \mathrm{e}-2$ \\
\hline Avg holding pattern & $1.63 \mathrm{e}+1 \pm 6.11 \mathrm{e}-1$ & $4.84 \mathrm{e}+1 \pm 1.29 \mathrm{e}+0$ \\
\hline & & \\
\hline std $=30 ;$ rotation & & \\
\hline Avg Waypoint spacing & $7.88 \mathrm{e}+0 \pm 9.53 \mathrm{e}-3$ & $7.85 \mathrm{e}+0 \pm 9.71 \mathrm{e}-3$ \\
\hline Avg holding pattern & 0 & 0 \\
\hline & & \\
\hline std = 60; rotation & & \\
\hline Avg Waypoint spacing & $7.59 \mathrm{e}+0 \pm 1.31 \mathrm{e}-2$ & $7.39 \mathrm{e}+0 \pm 1.30 \mathrm{e}-2$ \\
\hline Avg holding pattern & $5.48 \mathrm{e}-3 \pm 7.58 \mathrm{e}-3$ & $3.56 \mathrm{e}-2 \pm 2.87 \mathrm{e}-2$ \\
\hline
\end{tabular}

The major interest for this layout is the safety analysis in the next section, but a partial performance analysis was made. The case chosen is the long day where average aircraft arrival is 160 seconds and 500 aircraft arrive per day. Comparative statistics between the layouts for one year with a $95 \%$ confidence level are given in table 3 . 
Considering the results in table 3, Four Points and modified-Phoenix share four common elements: 1 runway, 4 Entry Points, 50 nautical miles from each Entry Point to the Waypoint, and at least 20 nautical miles from an Entry Point to the first junction. Until the results for rotation appeared, it seemed Four Points might be a universal example for arrival routes with these characteristics. On the other hand, this study was able to distinguish between the two. It also illustrates a general problem: given a pattern of aircraft arrival, what features of route layout are important.

\section{SAfety Analysis}

This section develops an approach for the statistical verification of a safety requirement. The major problem addressed is that a safety requirement is likely to be given for the entire airspace whereas any simulation is likely to model only a small part of air traffic operations. The question arises about what quantitative item an individual simulation should verify.

The safety criterion proposed in this section is hypothetical although it is hoped that it is similar enough to any criterion that might be proposed that the methods developed in this section are applicable.

This section considers scenarios that can be described as unsafe-events (UE). These events are not necessarily catastrophic events that result in the loss of life or property, but they can lead to catastrophic events. Unsafeevents require intervention, cause inconvenience, and are to be avoided.
Table 3. Comparison of layouts given average arrival time of 160 seconds.

\begin{tabular}{|l|l|l|}
\hline & Phoenix & Four Points \\
\hline \multicolumn{1}{|c|}{$s t d=30$} & & \\
\hline Avg Waypoint spacing & $7.89 \mathrm{e}+0 \pm 5.09 \mathrm{e}-3$ & $7.89 \mathrm{e}+0 \pm 9.80 \mathrm{e}-3$ \\
\hline Avg holding pattern & $1.80 \mathrm{e}+0 \pm 9.47 \mathrm{e}-2$ & $1.61 \mathrm{e}+0 \pm 1.96 \mathrm{e}-1$ \\
\hline & & \\
\hline \multicolumn{1}{|c|}{ std $=60$} & & \\
\hline Avg Waypoint spacing & $7.65 \mathrm{e}+0 \pm 1.50 \mathrm{e}-2$ & $7.65 \mathrm{e}+0 \pm 1.42 \mathrm{e}-2$ \\
\hline Avg holding pattern & $1.76 \mathrm{e}+1 \pm 6.32 \mathrm{e}-1$ & $1.63 \mathrm{e}+1 \pm 6.11 \mathrm{e}-1$ \\
\hline & & \\
\hline \multicolumn{1}{|c|}{ std $=30 ;$ rotation } & & \\
\hline Avg Waypoint spacing & $7.88 \mathrm{e}+0 \pm 4.99 \mathrm{e}-3$ & $7.88 \mathrm{e}+0 \pm 9.53 \mathrm{e}-3$ \\
\hline Avg holding pattern & $1.10 \mathrm{e}-2 \pm 5.46 \mathrm{e}-3$ & 0 \\
\hline & & \\
\hline \multicolumn{1}{|c|}{ std = 60; rotation } & & \\
\hline Avg Waypoint spacing & $7.60 \mathrm{e}+0 \pm 7.30 \mathrm{e}-3$ & $7.59 \mathrm{e}+0 \pm 1.31 \mathrm{e}-2$ \\
\hline Avg holding pattern & $1.19 \mathrm{e}+0 \pm 6.98 \mathrm{e}-2$ & $5.48 \mathrm{e}-3 \pm 7.58 \mathrm{e}-3$ \\
\hline
\end{tabular}

If experiments are performed locally for a global requirement, then it is necessary to combine the local confidence levels to obtain the overall confidence level. The necessary result is derived in [9]

Theorem: Suppose the parameter $\theta_{\mathrm{j}}$ has confidence interval $\left[\alpha_{\mathrm{j}}, \beta_{\mathrm{j}}\right]$ with confidence level $100\left(1-\mathrm{h}_{\mathrm{j}}\right) \%$. Then the vector parameter $\left(\theta_{1}, \theta_{2}, \ldots, \theta_{n}\right)$ has the vector confidence interval $\left(\left[\alpha_{1}, \beta_{1}\right],\left[\alpha_{2}, \beta_{2}\right], \ldots,\left[\alpha_{n}, \beta_{n}\right]\right)$ with an overall confidence level of $100\left(1-\mathrm{h}_{1}-\mathrm{h}_{2} \ldots-\mathrm{h}_{\mathrm{n}}\right) \%$.

Suppose the requirement considers only terminal operations and the requirement is less than an $\mathrm{R}$ chance that more than $\mathrm{Q}$ unsafe-events occur per year. Since the simulation only considers merging and interval management, we will let this part have the fraction $\mathrm{W}(\mathrm{W}<1)$ of the unsafe-events.

Hence, using the theorem above, we need to establish the safety of merging and interval management at the $100(1-$ WR) $\%$ confidence level.

We partition the universe of terminals by the percentage of aircraft that land at a terminal. Let $\mathrm{P}\left\{\mathrm{A}_{\mathrm{j}}\right\}$ be the probability that an aircraft lands at terminal $\mathrm{j}$, and let $\mathrm{P}\left\{\mathrm{UE}<\mathrm{W} \mid \mathrm{A}_{\mathrm{j}}\right\}$ be the conditional probability that there are less than $\mathrm{W}$ unsafe-events given an aircraft lands at terminal $\mathrm{j}$.

If there are $\mathrm{N}$ terminals, we write the probability of less than $\mathrm{W}$ unsafe events as

$$
\begin{aligned}
& \mathrm{P}\left\{\mathrm{A}_{1}\right\} \mathrm{P}\left\{\mathrm{UE}<\mathrm{W} \mid \mathrm{A}_{1}\right\}+\mathrm{P}\left\{\mathrm{A}_{2}\right\} \mathrm{P}\left\{\mathrm{UE}<\mathrm{W} \mid \mathrm{A}_{2}\right\}+\ldots+\mathrm{P}\left\{\mathrm{A}_{\mathrm{N}}\right\} \mathrm{P}\left\{\mathrm{UE}<\mathrm{W} \mid \mathrm{A}_{\mathrm{N}}\right\} \\
& \quad=\mathrm{P}\{\mathrm{UE}<\mathrm{W}\}
\end{aligned}
$$

By the theorem above, to establish this at the 100( $1-\mathrm{WR}) \%$ confidence level, we need to establish $\mathrm{P}\left\{\mathrm{UE}<\mathrm{W} \mid \mathrm{A}_{\mathrm{j}}\right\}$ at the $100\left(1-\mathrm{P}\left\{\mathrm{A}_{\mathrm{j}}\right\} \mathrm{W} \mathrm{R}\right\} \%$ confidence level. 
Note that considering only part of the entire air space operation imposes a penalty. First, the number of unsafe-events associated with merging and interval-management is only allowed to be W R whereas if the entire terminal was included in the simulation, all $\mathrm{R}$ of the unsafe-events could be associated with merging and spacing provided no other unsafe-events occurred

$\mathrm{E}_{4}$

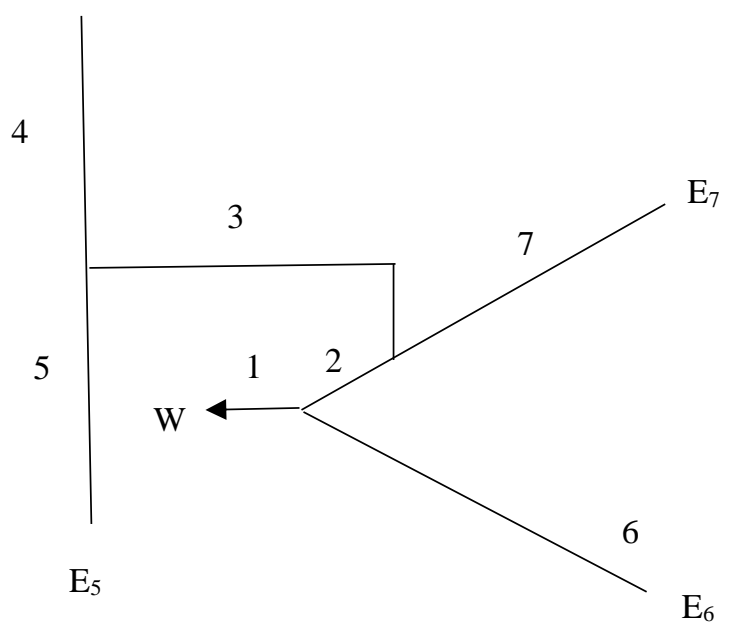

Figure 3: Standard terminal arrival routes based on Phoenix International at the terminal. Second, a high overall confidence level becomes numerous very high confidence levels as the verification is partitioned into smaller sections, and a very high confidence level requires numerous trials to establish. On the other hand, it does break the original problem into smaller pieces.

We will apply the above to a numerical example. Suppose the requirement focuses on only the terminals and it is that there be a less than $10 \%$ chance of more than 10 unsafe-events. Suppose merging and spacing is allowed to have $20 \%$ of the unsafe-events, and suppose there are 12 million flights per year and 500 flights per day land at the terminal under consideration. Then

$\mathrm{Q}=10$

$\mathrm{R}=10 \%$ or the overall confidence level is $100(1-\mathrm{R}) \%=90 \%$

$\mathrm{W}=0.2$

$\mathrm{P}\{\mathrm{A}\}=(500)(365) / 12000000=0.015$

Hence, we need to establish that there are two or less unsafe-events per year at the

$100(1-\mathrm{P}\{\mathrm{A}\} \mathrm{W} \mathrm{R}) \%=100(1-\mathrm{e}-4) \%$

confidence level.

Let $\mathrm{p}$ be the probability that more than two unsafe-events events occur at the terminal under consideration during a year. We want to establish that $\mathrm{p}<\mathrm{W} \mathrm{R}=0.02$ at the $100(1-\mathrm{e}-4) \%$ confidence level.

We will base our formulation on the observation that in preliminary runs no unsafe-event (loss of separation) has occurred. In this case, the number of trials $\mathrm{n}$ is given by

$$
(1-0.02)^{\mathrm{n}}=0.0001
$$

which gives $n=456$.

This number of trials was successfully performed for the Phoenix layout for the average arrival time of 160 seconds with a standard deviation of 60 seconds and no rotation. No loss of separation was observed for the simulated 456 years. Hence, there were never more than two losses of separation per trial.

The number of aircraft arrivals for the trials was (500) (365) (456) =83,220,000. It took a desktop computer running an interpretive language 106 hours to complete the trials.

\section{Summary}

The material above proposes a merging-and-spacing algorithm for an autonomous airport. This paper first performed a performance analysis for two layouts. It considered different distributions of arrival time, different distributions of Entry Points, and different operating times. It also examined a realistic layout based on the Phoenix Airport. A partial performance analysis was conducted for the Phoenix layout, but the emphasis was on a safety analysis. A key element for the safety analysis was determining what a local simulation should demonstrate when the safety requirement was given in terms of the global airspace.

Under all conditions, the simple algorithm developed in this paper was effective. It safely and efficiently handled all conditions of air traffic that were considered. The lacuna is that this initial effort lacked realism, and the consideration of additional scenarios has been postponed until more realism can be introduced.

The unique contribution of this paper is the factor analysis for performance and the statistical analysis for demonstrating safety at a sufficient confidence level.

\section{REFERENCES}

[1] L. Weitz, "A Fast-Time Study on Increasing the Capacity of Continuous Descent Approaches through Airborne Precision Spacing," These, Texas A\&M, August 2005. 
[2] D. Issacson, J. Robinson, H. Swenson, and D. Denery, “A Concept for Robust, High Density Terminal Air Traffic Operations," $10^{\text {th }}$ AIAA Aviation Technology, Integration, and Operations Conference, 13-15 September 2010, Forth Worth, Texas.

[3] J. Robison, D. Issacson, "A Concurrent Sequencing and Deconfliction Algorithm for Terminal Area Air Traffic Control," AIAA Guidance, Navigation, and Control Conference and Exhibit, 14-17 August 2000, Denver, CO.

[4] T. Nikoleris, H. Erberger, R. Paielli, and Y.-C. Chu, "Autonomous System for Air Traffic Control in Terminal Airsace," $14^{\text {th }}$ AIAA Aviation Technology, Integration, and Operations Conference, 16-20 June 2014, Atlanta, GA.

[5] R. Chipalkatty, P. Twu, A. Rahmani, and M. Egerstedt, "Distributed Scheduling for Air traffic Throughput Maximization During the Terminal Phase of Flight," at http://users.ece.gatech.edu/ magnus/Papers/ctre_CDC10_08_09_10.pdf.

[6] K. Swieringa, M. Underwood, B. Barmore, and R. Leonard, “An Evaluation of a Flight Deck Interval Management Algorithm including Delayed Target Trajectories," $14^{\text {th }}$ AIAA Aviation Technology, Integration, and Operations Conference, 16-20 June 2014, Atlanta, GA.

[7] B. Barmore, K. Swieringa, M. Underwood, T. Abbott, and R. Leonard, "Development of an Interval Management Algorithm using Ground Speed Feedback for Delayed Traffic," $14^{\text {th }}$ AIAA Aviation Technology, Integration, and Operations Conference, 16-20 June 2014, Atlanta, GA.

[8] J.-C. Ross, "Autonomous Take-Off and landing of a Low Cost Unmanned Aerial Vehicle," R\&D Journal f the South African Institute of Mechanical Engineering, vol. 2009, no. 25.

[9] K. Masuko and I Takahashi, “Autonomous Takeoff and Landing of an Unmanned Aerial Vehicle," IEEE/SICE International Symposium on System Integration, Sendai, 2010.

[10] A. White, N. Neogi, and H. Herencia-Zapana, "An Initial Examination for Verifying Separation Algorithms by Simulation,” Digital Avionics Systems Conference, Oct. 2012.

[11] Flight Operations Briefing Notes at HTTP://www.airbus.com/fileadmin/media_gallery/files/safety_library_items/AirbusSafetyLib-FLT_OPS-ApprSEQ03.pdf.

[12] S. Wilks, "Mathematical Statistics," Wiley, New York, 1968. 